\title{
Progress, Contribution and Challenges of Earth-Magnetism Navigation
}

\section{Liu Chang}

School of Automation and Electrical Engineering, University of Science \& Technology Beijing, Beijing, China

\author{
Email address: \\ xiangchang19920219@163.com
}

\section{To cite this article:}

Liu Chang. Progress, Contribution and Challenges of Earth-Magnetism Navigation. Automation, Control and Intelligent Systems. Vol. 5, No. 1, 2017, pp. 8-13. doi: 10.11648/j.acis.20170501.12

Received: December 2, 2016; Accepted: February 16, 2017; Published: March 3, 2017

\begin{abstract}
Navigation and positioning technology has stepped into the era of all schools of thoughts contend for attention. The progress, application, contribution and challenges of geomagnetic navigation technology are reviewed and analyzed. This paper introduces the principle and steps of geomagnetic navigation system, then compared with a variety of navigation technology to get the advantages and disadvantages of geomagnetic navigation; Summary of major contribution of geomagnetic navigation technology in the field of navigation and positioning; Finally briefly examine the important applications and the major challenges of geomagnetic navigation technology.
\end{abstract}

Keywords: Geomagnetic Navigation, Applications, Challenges

\section{Introduction}

This Earth's magnetic field is the inherent resources of the earth, which can be measured and be mapped, and provides a natural coordinate system for the aerospace, aviation and navigation. Geomagnetic navigation originated from orbit using the theory of Earth's magnetic field to determine the satellite's Psiaki other US Cornell University who proposed the theory since 1989, geomagnetic field navigation has become a major research focus [1]; In 1960s saw the emergence of the earliest geomagnetic navigation experiment, USA E-system company developed abnormal contour matching system based on geomagnetism, and in the 1970s, the use of geomagnetic anomaly data was carried out off-line experiments [2]; in 1980s, geomagnetism US military navigation systems as the key technologies. In addition to the United States, other countries are also do a lot of research on the geomagnetic navigation, such as Sweden's Carl utilize geomagnetic for vessels navigating programs to achieve the measurement of the position and speed of the vessel [3] [4]; the French used magnetic guidance system Research on a new type of ammunition, and achieved good effects in tests in guidance; The Raytheon company successfully achieved by using a magnetic field experimental underground navigation [5]. In 2006, Felix Goldenberg inertial aircraft / GPS / geomagnetic matching navigation system is studied, and analyzed using a three-axis magnetometer measured separation process magnetic anomaly field on the plane, the plane proved on even the possibility of the use of space geomagnetic navigation [6]. Meanwhile, in the 1980s, underwater geomagnetic navigation technology began to rise. In 1986, Westinghouse Electric Company presents a stationary magnetic field with known exceptions to the underwater vehicle navigation method [7]. 1987-1989, Sweden completed a feasibility study of underwater geomagnetic navigation, and developed a special geomagnetic sensor [8]. The study abroad for geomagnetic navigation has established a mature system, technology is more perfect.

In contrast the geomagnetic navigation development at home, although we have to start very early ancestors of using a magnetic field for navigation, such as the compass, but the research and application of modern magnetic navigation technology is rather late, and most of them stay in the theoretical stage. In the 21 st century, it has been found valuable research results. In 2009 University of Defense Technology $\mathrm{Hu}$ Zhengdong has applied unscented Kalman filter algorithm to geomagnetic navigation, and by comparing the simulation, which is better than extended Kalman filter navigation performance [9]; In 2010, University of Science \& Technology Beijing Li Xisheng proposed based on ICCP Algorithm geomagnetic matching technology research, 
through the improvement to the algorithm improves the geomagnetic matching accuracy and rapidity [10].

In recent years, through the joint efforts of countries in the field of navigation researchers geomagnetic navigation at the technical level has made great strides.

Geomagnetic navigation technology has better compatibility and interoperability then other navigation systems. Compatibility means separate or integrated use different navigation systems, will not cause unacceptable interference, a navigation system cannot cause harm to the service; interoperability refers geomagnetic navigation technology can combine with other navigation techniques to enhance the service capabilities of the system, it has better capacity than a single service at the user level. Clearly, compatibility is based on interoperability [11].

Of course, there are some disadvantages of geomagnetic navigation, and it is greatly influenced by the living environment changes in the city. The establishment of database requires a lot of manpower and material resources and other issues; Geomagnetic navigation technology also faced with competition and other satellite navigation market problems.

In this paper, a brief introduction on the basis of the principle of a geomagnetic navigation system and the establishment of principles, with emphasis on a magnetic navigation technology and its contribution in the field of navigation; at the same time, based on the relevant literature reading and understanding of the author, but also a simple analysis of geomagnetic navigation technical problems faced.

\section{Establish Principles and Steps of Geomagnetic Navigation System}

The Geomagnetic navigation feature is the use of Earth's magnetic field for navigation method, the main way is divided into geomagnetism and geomagnetic matched filtering, the first using more. In fact, geomagnetism matching navigation essence is the digital map-matching algorithm on. Geomagnetic matching based on the Earth's magnetic field is a vector field, which is at any point in the near-Earth region of the geomagnetic field characteristics are different, and one relationship exists with the latitude and longitude of the point. Theoretically, you can create a database of the geomagnetic field, which can be established to measured magnetic field characteristics of a point to use data matching techniques to complete the positioning work [13].

\section{Compared with Other Navigation Technology}

Earth's magnetic field is a vector field (including North component east component and the vertical component of the total field vector synthesis), the global distribution of near-Earth space continuously. It varies with time and space changes. Modern geomagnetism theory that Earth's magnetic field strong field consists of three parts, namely:

$$
B(R, T)=B_{m}(R, T)+B_{a}(R)+B_{d}(R, T)
$$

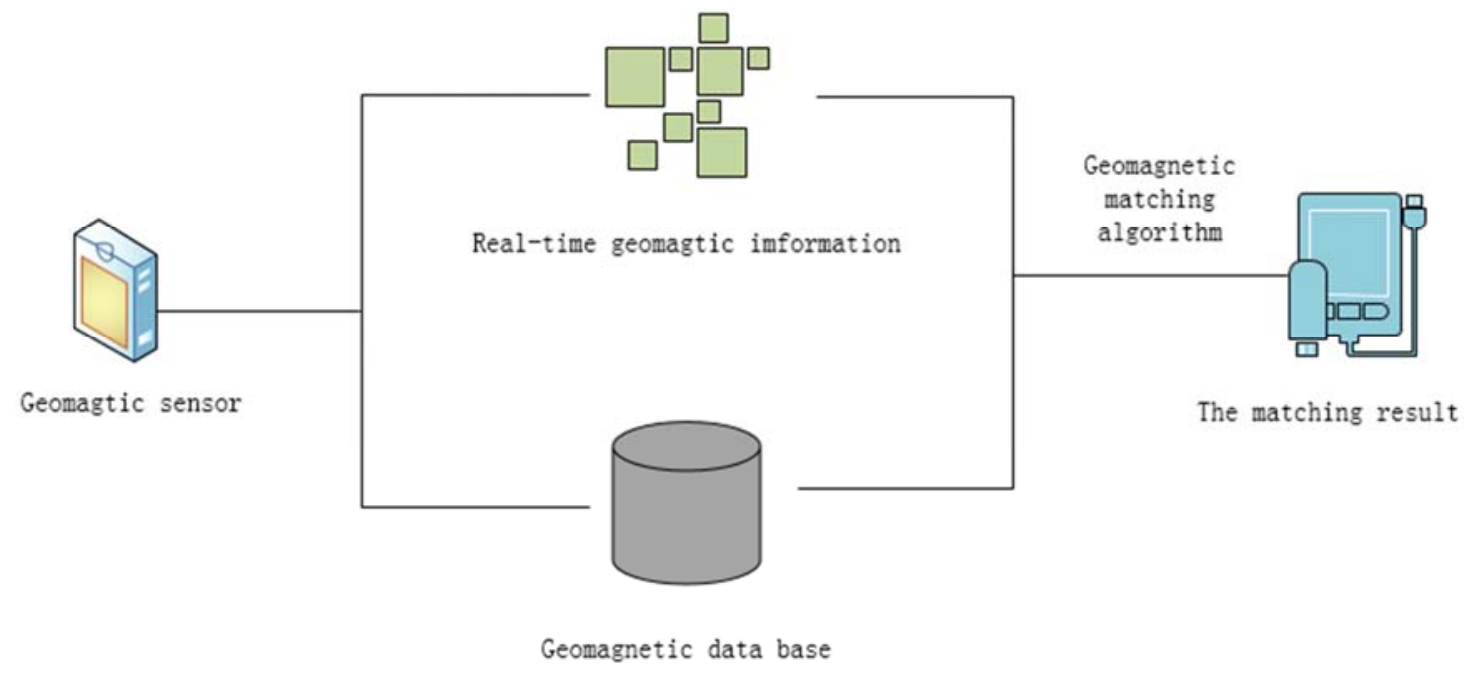

Figure 1. Schematic geomagnetic match.

In the formula, $B_{m}(R, T)$ is the main magnetic field (also known as core field), is under the mantle, the outer core of liquid iron and nickel circulation caused by high temperatures, change slowly over time, the magnitude of change of global average annual 80nT; spatial distribution of planetary scale; in strength at the surface is $50000 \sim 70000 \mathrm{nT}$, covering more than 95 percent of the total magnetic field. $B_{a}(R)$ Abnormal field (also called the crust field), resulting in the magnetization of crustal rocks, almost no change over time, the spatial distribution of rich frequency, wavelength, from one thousand meters to several thousand meters, in $4 \%$ of the total magnetic field strength of the surface area of at least; $B_{d}(R, T)$ To interfere with the magnetic field from the magnetosphere and ionosphere, contains both the diurnal variation and change of rules such as interference, but also contains storms, sub-storms and other irregular interference, 
non-magnetic interference during storms typically tens Nath, when magnetic storms can exceed 100nT. Geomagnetic matching navigation is mainly used inside the Earth's core and crust field, the core field and crustal field is a major part of the geomagnetic field, changes slowly over time, has become a stable magnetic field which is suitable for geomagnetic matching navigation technology.

Geomagnetic navigation feature is the use of Earth's magnetic field for navigation method, the main way into geomagnetism and geomagnetic matching filter, the geomagnetism matching using more, the essence of geomagnetic matching navigation is the digital map-matching algorithm. Geomagnetic matching based on the Earth's magnetic field at any point in the near-Earth region of the geomagnetic is a vector field, and the field characteristics are different, which shows the relationship exists with the latitude and longitude of the point.
Theoretically, you can create a database of the geomagnetic field, the measured magnetic field characteristics of a point to use data matching techniques to complete the positioning work [14].

Matching a simple correlation algorithm considered to meet the criteria related to certain points is by seeking common criteria and conditions related to the Table1:

Among them: $M_{M}^{k}$ Earth's magnetic field strength of the magnetic sensor, $M_{S}^{k}$ Corresponding to the location of the Earth's magnetic field vector strength, $\mathrm{N}$ is the number of samples geomagnetic worth.

In the table, the PROD and the NPROD emphasize that the similarity between the two objects, while the MAD and the MSD emphasize the degree of difference between the two objects. The four algorithms are widely used in the correlation analysis, the MSD is accurate relatively.

Table 1. Correlation criteria and conditions.

\begin{tabular}{llc}
\hline Correlation metric algorithm & Formula & Best match metric \\
\hline Product correlation algorithm PROD & $\operatorname{prod}_{i j}=\frac{1}{N} \sum_{k=1}^{N} M_{S(i, j)}^{k} M_{M}^{k}$ & Max \\
Normalized product correlation algorithm NPROD & $\operatorname{nprod}_{i j}=\frac{\sum_{k=1}^{N} M_{S(i, j)}^{k} M_{M}^{k}}{\left[\sum_{k=1}^{N} M_{S(i, j)}^{k}\right]^{\frac{1}{2}}\left[\sum_{k=1}^{N} M_{M}^{k}\right]^{\frac{1}{2}}}$ & Max \\
MAD - Mean absolute deviation & $\operatorname{mad}_{i, j}=\frac{1}{N} \sum_{k=1}^{N}\left|M_{S(i, j)}^{k}-M_{M}^{k}\right|$ & Min \\
Mean variance algorithm MSD & $\operatorname{msd}_{i, j}=\frac{1}{N} \sum_{k=1}^{N}\left(M_{S(i, j)}^{k}-M_{M}^{k}\right)^{2}$ & Min \\
\hline
\end{tabular}

Table 2. Comparison of three main navigation technology.

\begin{tabular}{|c|c|c|c|c|c|}
\hline $\begin{array}{ll}\text { Navigation } & \text { Comparative aspects } \\
\end{array}$ & Active or not & autonomous navigation & interference & Have accumulated error & Accuracy \\
\hline Earth-Magnetism navigation & $x$ & $\sqrt{ }$ & $\sqrt{ }$ & $x$ & Moderate \\
\hline Inertial guidance & $\sqrt{ }$ & $\sqrt{ }$ & $\sqrt{ }$ & $\sqrt{ }$ & Low \\
\hline GPS navigation & $\sqrt{ }$ & $x$ & $\times$ & $x$ & High \\
\hline Doppler navigation & $\sqrt{ }$ & $\times$ & $\times$ & $\sqrt{ }$ & High \\
\hline
\end{tabular}

Feature matching is a key technology for in the field of image and vision. The key is choosing the right feature matching feature amount. Matching the feature quantity selection needs to consider a long-term feature amount geomagnetic field changes the degree of stability, short-term changes in the degree of relevance with the location, performance and feature information matching area existing measurement equipment and other factors [15], feature amount geomagnetic field can be divided into two categories, one is the strength categories, including overall strength, the strength of the east, north intensity, horizontal intensity and vertical intensity; the other is the perspective of the class, including the declination and inclination. To achieve geomagnetic match, we need to match the region feature amount sufficient feature information, which can be mathematically described as a feature amount statistical characteristics in a matching area, and the indirect expression is the application condition of the matching algorithm.
Geomagnetic matching, it would normally select a feature amount as the matching characteristics.

Modern navigation and positioning technology presents a wide range of trends, and the main navigations include inertial navigation, GPS navigation [16] and Doppler navigation [17]. Compare these three navigation technology from the products cost, positioning accuracy, power size, interference, etc., have their own advantages and disadvantages. (as shown in the Table 2)

As can be seen from Table 2, the main navigation techniques have their own advantages and disadvantages, such as the geomagnetic navigation, although passive immunity passive navigation, and has the advantages of anti-interference and non-cumulative error, but its accuracy is not high enough, often fail to project requirements; at the same time, GPS navigation technology has high-precision, but not interference; it can be seen from both navigation between the two complementary, thus taking into account the 
two or more navigation technology integration, mutual correction, to get more precise positioning and navigation results.

Geomagnetic navigation features make it to be an important compensation and to correct other navigation technology, it is an important part of SINS. It works as figure 2:

\section{The Important Problem of the Applications of Magnetic Navigation}

The progress and development of the geomagnetic measurement techniques, so that the advantages of geomagnetic navigation theory have become an increasingly prominent, as a new passive navigation, geomagnetic navigation can achieve all-weather autonomous navigation. This navigation has a good hiding, ready to use, no accumulated error, etc., can be used for autonomous navigation and remote-guided missiles and other weapons, submarines, ships, vehicles and other carriers, it has shown significant military value and prospect [18]. In addition the use of geomagnetic matching navigation composition strap down navigation and other navigation can overcome the shortcomings of single navigation mode, in order to achieve a long and highly accurate navigation.
At present, domestic and foreign scholars geomagnetic navigation and navigation algorithm implementation has done a lot of researches, and the use of field measurements data simulation experiments, and made many meaningful results, however, there are still three big mountains strong geomagnetic which restrict the development and application of matching navigation.

\subsection{Establishing an Accurate Model}

Establishing an accurate model of the geomagnetic field is referenced navigation positioning, the geomagnetic field model is divided into global and regional geomagnetic field model geomagnetic field model, the existing global geomagnetic field model is only part of the description of the main magnetic field, a limited precision, and can not reflect the complexity geomagnetic anomalies. So in precise navigation requirements, we need a local geomagnetic field model. Furthermore, since the earth's magnetic field characteristic quantities susceptible to external influence, such as Earth's magnetic field with a high degree of variation of the geomagnetic field and the fluctuation rule, these problems are currently no definitive conclusions, further research is needed to explore.

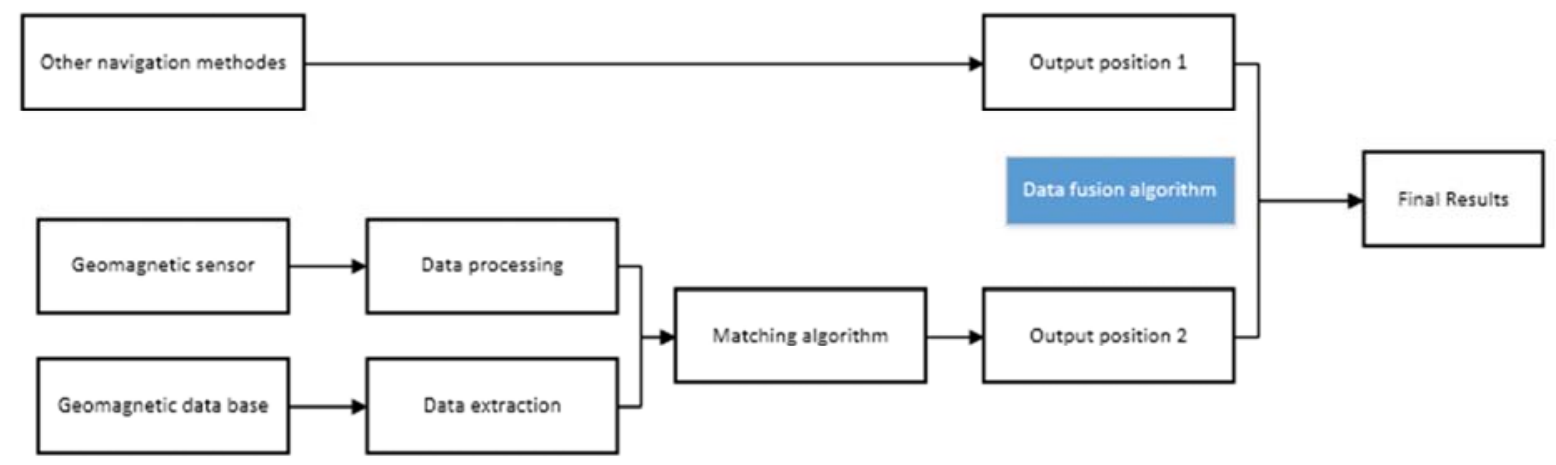

Figure 2. Navigation schematic.

\subsection{Performance Measurement of Magnetic Instruments and the Measured Information Processing}

Want to take advantage of geomagnetic matching navigation method, first detected magnetic feature information, and to obtain accurate geomagnetic feature information, the need for high performance magnetic measurement instruments, in addition to the need from the measured values of various excluded interference, etc., which need to have appropriate technology as a support, such as magnetic interference cancellation techniques, error compensation technology. It has developed a variety of high precision sensors for measuring weak magnetic geomagnetic information, but there are no effective techniques for interference cancellation and error compensation.

\subsection{Study Geomagnetic Matching Algorithm}

Geomagnetic matching algorithm determines the precise geomagnetic matching navigation key factor in whether or not the geomagnetic matching algorithm of the key technical issues become a major issue facing the geomagnetic navigation. Read the relevant literature, are summarized as follows:

\subsubsection{Algorithm to Ensure Uniqueness of the Geomagnetic Matching Results}

The unique compared results is to ensure the accuracy of matches, if the match is not the only result will be greatly reduced or even result in completely deviated from the navigation precision navigation This is the first problem of geomagnetic matching method need to be solved.

\subsubsection{Matching Algorithm to Be Taking into Account the Accuracy and Timeliness}

Geomagnetic matching algorithm is necessary to consider certain environment matching accuracy, but also consider the degree of complexity of the algorithm and computation time, if the algorithm is too complicated, the operation time is too 
long, so that real-time navigation of the greatly reduced. Matching algorithm requires both a broad global search capability, but also a precise local positioning capability [19].

\subsubsection{Application of Geomagnetic Navigation Need to Distinguish Between Indoor and Outdoor Environments}

Geomagnetic environment within the building compared to the open outdoor environment, factors to be considered very complex and includes not only the magnetic field to the stadium itself, but also includes a magnetic field inside a building due to the factor of building materials, metal doors and windows as well as indoor and other iron objects produced, collectively referred to as an additional field.

Additional farm buildings are the result of the combined effect of a variety of magnetic factors, the entire building is non-uniform, modern buildings are made of reinforced concrete or steel construction made under the influence of the geomagnetic field, because is magnetized to produce a significant magnetic field characteristics and location of these features can be as part of an indoor positioning and navigation data source.

Relevant literature shows that additional field also has differences and stability in different locations on time, it can be used as a basis to stab navigation.

\subsubsection{Geomagnetic Navigation Problems in the Practical Application}

There are many skyscrapers in the modern city, within various buildings contain rich ferromagnetic material, such as steel, pipes, etc., which resulted in the building of a strong magnetic field disturbance. In terms of location algorithm, if used geomagnetic positioning signal, the need for calibration, which will affect the positioning speed, in general, geomagnetic positioned to assist other technical.

\section{Conclusion}

Geomagnetic navigation technology with its unique performance and rapid pace of development, to accomplish something in the military field, especially in the low-altitude areas of guidance, but in order to make a real geomagnetic navigation technology into the civilian era, there is still a long way to go. From the current market situation, although there are many products on the market claim to have pedestrian navigation features, these products are mobile phone, PDA or tablet PC represented, but the main positioning technology are limited to either rely solely on GPS positioning, or through mobile wireless base station location, there are some devices using map matching algorithm assisted GPS and Wi-Fi positioning (GPS / MM). However, the reality is that more and more intensive modern urban construction, building more and more high buildings, viaducts, tunnels criss-cross, in the subway, indoor and other GPS signals are seriously blocked areas, it is difficult to provide continuous and accurate GPS navigation information, even in the outdoors, often due to the reflection of signals between towers so that GPS signals cause the affected navigation accuracy is not high enough to fully meet the needs of real-time navigation information and the accuracy of the pedestrian navigation system.

In recent years, with the establishment of large-scale urban tunnels, overpasses and other buildings which have a height difference, the satellite navigation "urban effect" has become more evident, no doubt to the strap down navigation field bring some opportunities, but at the same time, both opportunities and challenges, how to gradually improve and geomagnetic navigation technology to enable it to make up for the satellite navigation shortcomings, as we become a serious problem.

However it is expected that the majority of our navigation through the joint efforts of scientists, geomagnetic navigation technology is expected to achieve new breakthroughs. And international navigation in the field have made outstanding contributions to the future!

\section{References}

[1] YANG Gong-liu LI Shi-xin, JANG Chao-yu. Data fusing algorithm in geomagnetic aided INS [J]. Journal of Chinese Inertial Technology, 2007, 5 (1): 46-49

[2] Goldenberg F. Geomagnetic navigation beyond the magnetic compass [C]//2006 IEEE/ION Position, Location, And Navigation Symposium. IEEE, 2006: 684-694.

[3] Tyren C. Magnetic anomalies as a reference for ground-speed and map-matching navigation [J]. Journal of Navigation, 1982, 35 (02): 242-254.

[4] Merrill M L. Unmanned untethered submersible technology [J] Marine Technology Reporter, 2007, 50 (8).

[5] Davis C. GPS-like navigation underground[C]//Proceedings of IEEE/ION PLANS 2010. 2001: 1108-1111.

[6] Hasan A M, Samsudin K, Ramli A R, eta/. A review of navigation systems (inlegrationand algorithms). Australian journal of Basicand Applied Sciences, 2009; 3 (2): 943-959

[7] Polvani D. Magnetic guidance of autonomous vehicles (part 2) $[\mathrm{C}] / /$ Unmanned Untethered Submersible Technology, Proceedings of the 19875 th International Symposium on. IEEE, 1987, 5: 257-264.

[8] Xiao Yijun, Ding Mingyue, Zhou Chengping. Matching suitability analysis of reference map for scene matching based navigation. International Symposiumon Multispectrol Image Processing, SPIE Vol. 3545: 100 103.

[9] Winter S, Pontikakis E, Raubal M. LBS for Real-Time Navigation-A Scenario [J]. GeoInformatics, 2001, 4 (4): 6-9.

[10] Sagawa K, Inooka H, Satoh Y. Non-restricted measurement of walking distance $[\mathrm{C}] / /$ Systems, Man, and Cybernetics, 2000 IEEE International Conference on. IEEE, 2000, 3: 1847-1852.

[11] Akasaka Y, Onisawa T. Construction of pedestrian navigation system and its evaluation [C]//Fuzzy Systems, 2004. Proceedings. 2004 IEEE International Conference on. IEEE, 2004, 3: $1525-1530$.

[12] Ailisto H J, Lindholm M, Mantyjarvi J, et al. Identifying people from gait pattern with accelerometers [C]//Defense and Security. International Society for Optics and Photonics, 2005: 7-14. 
[13] Zhao Jianhu. Study on underwater navigation system based on geomagnetic match technique. The Ninth International Conference on Electronic Measurement \& Instruments.2009.

[14] Xinlong Jiao, Xue lian Liu. Model of quantitative evaluation of logistics enterprise customer satisfaction [A]. Proceedings of 2014 International Conference on Information Management and Management Engineering (IMME 2014) [C]. 2014.

[15] Shuai Huang 1, Shuyan Sun 1, Guanhong Tao 1 and Jiankang Wu 1,2 1 Sensor Networks and Applications Joint Research Center (SNARC) Graduate University and Institute of Automation, Chinese Academy of Sciences 2 China-Singapore Institute of Digital Media (CSIDM) Beijing, China. Calibration of a Magnetic and Inertial Measurement Unit without Additional Equipment [A]. IEEE. Proceedings of 2011 4th IEEE International Conference on Computer Science and Information Technology (ICCSIT2011) OL02[C]. IEEE:, 2011:6.

[16] Alkan R M, Karaman H, Sahin M. GPS, GALILEO and GLONASS satellite navigation systems \& GPS modernization[C]/Proceedings of 2nd International Conference on Recent Advances in Space Technologies, 2005. RAST 2005. IEEE, 2005: 390-394.

[17] Lee P M, Kim S M, Jeon B H, et al. Improvement on an inertial-Doppler navigation system of underwater vehicles using a complementary range sonar[C]//Underwater Technology, 2004. UT'04. 2004 International Symposium on. IEEE, 2004: 133-138.

[18] Lin Xueyuan Chen Yulin Zhou Min (Department of Electronic, Naval Aeronautical Engineering Institute, 188 Erma Street, Yantai 264001, China). Error Analysis and Compensation of Three-axis Strap-down Magnetometers [A]. Chinese Institute of Electronics (CIE). Proceedings of 2007 8th International Conference on Electronic Measurement \& Instruments (ICEMI'2007) Vol. I [C]. Chinese Institute of Electronics (CIE):, 2007:4

[19] Gadre AS. Stilwell D J. Toward underwater navigation based on range measurements fromasingle location. In: Proceedings of 2004IEEE International Conference on Robotics and Automation (ICRA04), 2004; 5: 4472-----4477
[20] Weinan Xie, Zhenshen Qu, Qinghua Li. A Fast Algorithm of the Geomagnetic Correlation Matching Based on MSD [A]. American Applied Sciences Research Institute, AASRI. P roceedings of 2013 Third International Conference on Control, Automation and Systems Engineering (CASE 2013) [C]. American Applied Sciences Research Institute, AASRI:, 2013:4.

[21] Rong L, Jianzhong Z, Ming L, et al. A wearable acceleration sensor system for gait recognition[C]//2007 2nd IEEE Conference on Industrial Electronics and Applications. IEEE, 2007: 2654-2659.

[22] He Z, Jin L. Activity recognition from acceleration data based on discrete consine transform and SVM[C]//Systems, Man and Cybernetics, 2009. SMC 2009. IEEE International Conference on. IEEE, 2009: 5041-5044.

[23] LIU Fei, ZHOU Xian-gao, YANG Hua, et al. Geomagnetic matching location using correlative method [J]. Journal of Chinese Inertial Technology, 2007, 15 (1): 59-62.

[24] Nikolai Petersen. Biomine ralization and magnetism of bacterial magnetosomes [J]. Chinese Science Bulletin, 2004, 24: 2563-2568.

[25] Nowicki M, Szewczyk R. Ferromagnetic Objects Magnetovision Detection System [J]. Materials, 2013, 6(12): 5593-5601. Chung J, Donahoe M, Schmandt C, et al. Indoor location sensing using geo-magnetism[C]/Proceedings of the 9th international conference on Mobile systems, applications, and services. ACM, 2011: 141-154.

[26] Gozick B, Subbu K P, Dantu R, et al. Magnetic maps for indoor navigation $[\mathrm{J}]$. IEEE Transactions on Instrumentation and Measurement, 2011, 60(12): 3883-3891.

[27] Le Grand E, Thrun S. 3-axis magnetic field mapping and fusion for indoor localization[C]//Multisensor Fusion and Integration for Intelligent Systems (MFI), 2012 IEEE Conference on. IEEE, 2012: 358-364. 